\title{
Comparative genomic analysis of the secondary flagellar (flag-2) system in the order Enterobacterales
}

\author{
Pieter De Maayer ${ }^{1 *}$ (D), Talia Pillay ${ }^{1}$ and Teresa A. Coutinho ${ }^{2}$
}

\begin{abstract}
Background: The order Enterobacterales encompasses a broad range of metabolically and ecologically versatile bacterial taxa, most of which are motile by means of peritrichous flagella. Flagellar biosynthesis has been linked to a primary flagella locus, flag-1, encompassing 50 genes. A discrete locus, flag-2, encoding a distinct flagellar system, has been observed in a limited number of enterobacterial taxa, but its function remains largely uncharacterized.

Results: Comparative genomic analyses showed that orthologous flag-2 loci are present in 592/4028 taxa belonging to 5/8 and 31/76 families and genera, respectively, in the order Enterobacterales. Furthermore, the presence of only the outermost flag-2 genes in many taxa suggests that this locus was far more prevalent and has subsequently been lost through gene deletion events. The flag-2 loci range in size from $~ 3.4$ to 81.1 kilobases and code for between five and 102 distinct proteins. The discrepancy in size and protein number can be attributed to the presence of cargo gene islands within the loci. Evolutionary analyses revealed a complex evolutionary history for the flag-2 loci, representing ancestral elements in some taxa, while showing evidence of recent horizontal acquisition in other enterobacteria.

Conclusions: The flag-2 flagellar system is a fairly common, but highly variable feature among members of the Enterobacterales. Given the energetic burden of flagellar biosynthesis and functioning, the prevalence of a second flagellar system suggests it plays important biological roles in the enterobacteria and we postulate on its potential role as locomotory organ or as secretion system.
\end{abstract}

Keywords: Enterobacterales, flag-2, primary and secondary flagellar system, Flagellin glycosylation, Motility

\section{Background}

The order Enterobacterales encompasses a diverse group of Gram-negative, non-sporing, facultatively anaerobic rod-shaped bacteria. Recent phylogenomic re-evaluation of the sole family in this order, the Enterobacteriaceae, has resulted in its division into eight distinct families [1]. Members of this order can be found in a diverse range of environments including air, soil, water and in association with plant and animal hosts, and include some of the most important pathogens of these hosts [2]. Key to the ecological success of enterobacteria is their capacity for motility, which is largely mediated by flagella, specialized surface structures that allow bacterial cells to

\footnotetext{
* Correspondence: Pieter.Demaayer@wits.ac.za

${ }^{1}$ School of Molecular \& Cell Biology, University of the Witwatersrand, 2050

Wits, Johannesburg, South Africa

Full list of author information is available at the end of the article
}

move along surfaces, towards nutrients and away from harmful substances [3]. Furthermore, flagella play crucial roles in enterobacterial pathogenesis, contributing to adherence, invasion and colonization of host cells and tissues $[4,5]$.

Flagella are highly complex structures, comprised of three major components, a basal body, hook and filament [6]. The basal body anchors the flagellum to the cell envelope and incorporates the flagellar motor $[3,6]$. The flagellar hook connects the basal body to the flagellar filament and acts as a universal joint, facilitating dynamic and efficient motility and taxis [7, 8]. The filament is the longest, surface-exposed, component of the bacterial flagellum and is composed of approximately 20,000 subunits of the major structural protein $[6,9]$. This filament serves as a propeller, which converts the motor into thrust to propel the bacterial cell [9].

(c) The Author(s). 2020 Open Access This article is distributed under the terms of the Creative Commons Attribution 4.0 International License (http://creativecommons.org/licenses/by/4.0/), which permits unrestricted use, distribution, and 
Typically, up to 50 genes are required for the assembly, maintenance and functioning of these surface appendages [10]. In the model enterobacterial taxa Escherichia coli and Salmonella enterica, the genes involved in flagellar biosynthesis and functioning are located in three genomic clusters, collectively termed the primary flagellar locus (flag-1) [11, 12]. Although most of the genes involved in flagellar biosynthesis are common to most bacterial taxa, a high level of divergence in flagellar structure exists and allows different microorganisms to be distinguished from one another [10]. Furthermore, flagellin glycosylation and methylation has been observed in a number of bacterial species and has shown to play a crucial role in flagella assembly and virulence $[13,14]$.

In addition to the primary flagellar system, a number of enterobacterial taxa, namely E. coli, Yersinia enterocolitica, Yersinia pestis and Citrobacter rodentium, have been observed to possess a distinct secondary flagellar (flag-2) system $[15,16]$. This flag-2 system has been attributed to a specific genomic locus, which resembles that coding for the lateral flagella in Aeromonas hydrophila and Vibrio parahaemolyticus, and is genetically distinct from the gene clusters that are required for the biosynthesis of the primary flagellar system [15]. The flag-2 locus of E. coli 042 is $\sim 48.8 \mathrm{~kb}$ in size and codes for 44 distinct proteins involved in the synthesis. This second flagellar system has been suggested to facilitate swarming motility on solid surfaces [15]. Knock-out mutagenesis of the $Y$. enterocolitica flag-2 system, however, had no effect on motility, and it was suggested to serve as a virulence factor that aids this pathogen gain entry into mammalian cells [16]. Here, by means of comparative genomic analyses, we have further analysed the flag2 locus and show it to present in a substantial number of taxa across a broad spectrum of the genera and families in the order Enterobacterales. The enterobacterial flag-2 locus comprises a large set of conserved genes for the synthesis and functioning of the secondary flagellar system, but also incorporates variable regions that may contribute to both structural and functional versatility of this system. Our genomic analysis suggests that the flag2 locus may have been universally present in some enterobacterial lineages, and that this ancestral locus has subsequently been lost in some taxa, while in other lineages it has been derived through horizontal gene acquisition. Finally, we postulate on the potential functions of this versatile and widespread flagellar system in the Enterobacterales.

\section{Results and discussion}

\section{The flag-2 locus is widespread among the} Enterobacterales

The finished and draft genomes of 4028 bacterial strains encompassing the taxonomic diversity of the order
Enterobacterales were screened for the presence of flag2 loci (Additional file 1: Table S1). A total of 592 (15\% of the analysed taxa) strains were observed to possess an orthologous locus (Fig. 1 - indicated by green circles; Additional file 1: Table S1) and these are distributed across a wide taxonomic breadth of the order. As such, flag-2 loci occur in five of the eight families and 31/76 genera included in this study. Exceptions are observed for the families Morganellaceae (7 genera - 313 strains), Pectobacteriaceae (7 genera - 244 strains) and Thorselliaceae (Thorsellia - 1 strain), where no flag-2 loci occur. The highest prevalence can be observed in the family Budvicaceae (7/9 studied taxa) and Yersiniaceae (225/605 strains), while only $13 \%$ (316/2464 taxa) of the family Enterobacteriaceae contained orthologous loci (Fig. 1; Table 1). Differences in prevalence at the genus level could also be observed. Notably, flag-2 loci are universally present in several genera, including Citrobacter Clade D (30/30 strains) and Plesiomonas (8/8 strains), while in the two genera with the highest number of flag2 loci present, Yersinia (222/394 strains) and Escherichia (124/522 strains), 56 and $24 \%$ of the evaluated strains encode flag- 2 systems, respectively. In some genera, the presence of flag-2 loci represents a rare trait. For example, only two of 151 analysed Pantoea strains contain flag-2 loci. Diversity in terms of flag-2 locus presence can furthermore be observed at the species level. For example, all 100 of the evaluated $Y$. pestis strains incorporate a flag-2 locus, while it only occurs in $24 / 100$ Escherichia coli strains.

\section{Molecular architecture of the flag-2 loci}

The flag-2 loci comprise of a set of co-localised genes within the genomes of the enterobacteria that harbour them (Fig. 2). This is in contrast to the flag-1 system (Fig. 3), where gene loci responsible for the synthesis and functioning of the primary flagellar system are generally dispersed across the enterobacterial chromosome [12]. The enterobacterial flag-2 loci range in size from 3.4 to $\sim 81.8$ kilobases (average $38.1 \mathrm{~kb}$ ) and code for between five and 102 (average 43 proteins) proteins (Additional file 1: Table S2). The discrepancy in size and number of proteins encoded by the loci can largely be attributed to frequent deletions and insertion of noncore genes within the loci. Substantially larger flag-2 loci are observed in Escherichia albertii B156, Citrobacter (Clade A) sp. nov $1 \mathrm{~S} 1285$ and three C. rodentium strains. This can be linked to the insertion of prophage elements within the flag-2 loci, contributing on average $37.7 \mathrm{~kb}$ of sequence and 54 proteins.

Comparative analysis showed extensive synteny and sequence conservation among the flag-2 loci (Fig. 2). Of the 592 strains with flag-2 loci, 461 (77.87\% of strains with flag-2 loci) encode an orthologue complement of 


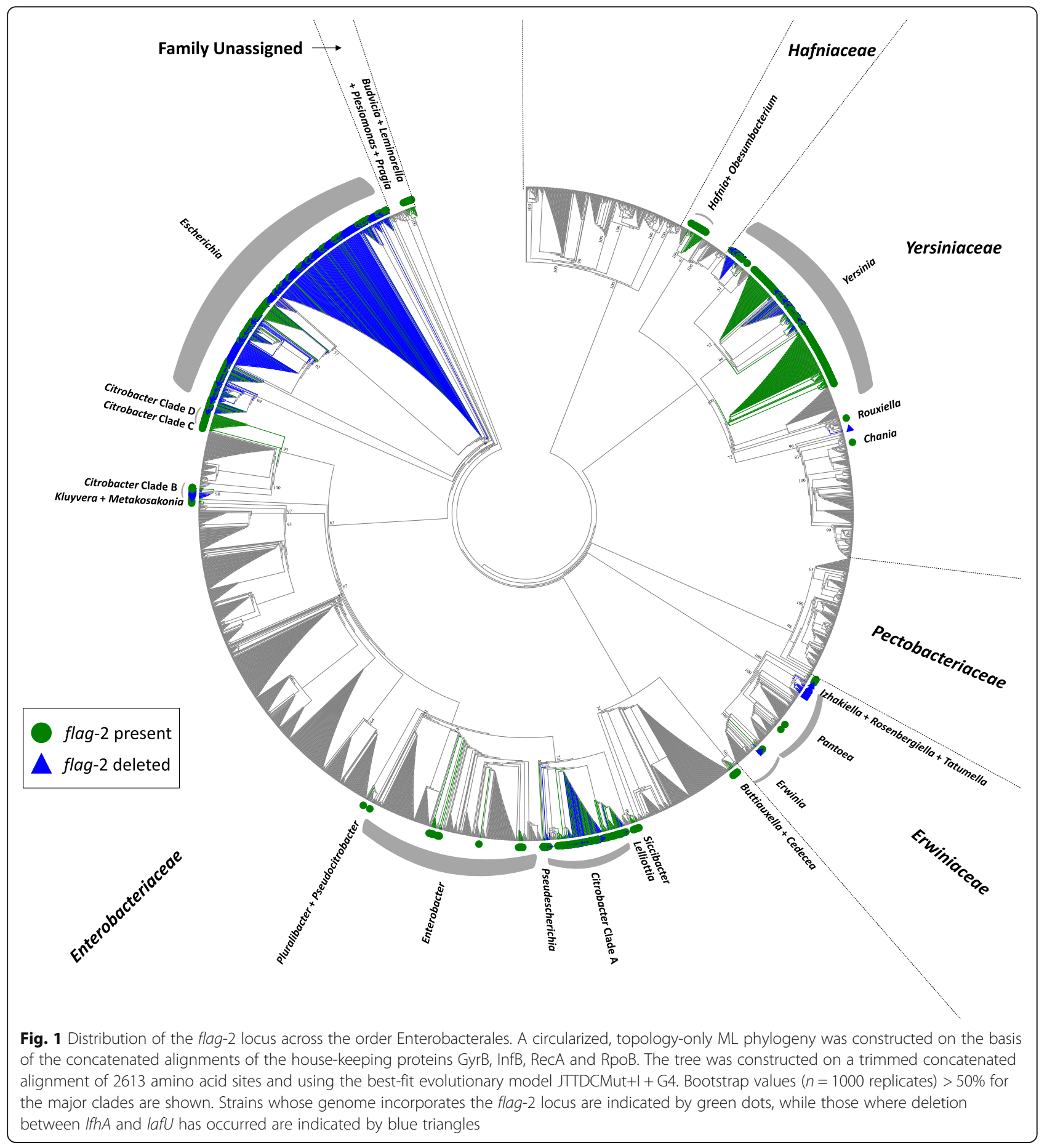

39 conserved proteins. One of these conserved proteins is LafA, the flagellin counterpart of the flag- 2 system, which is present in multiple copies in 156/592 (26.35\%) strains, with up to five copies ( $Y$. pestis Pestoides B $81.81 \%$ average amino acid identity) encoded by the flag2 locus. Multiple copies of the flagellin gene have also been observed in the flag-1 loci of many enterobacteria and have been suggested to contribute to the phenomenon of phase variation [14, 17, 18]. As flagellin proteins are potent antigens, the phase variable expression of these proteins may enable these organisms to temporarily avoid immune responses in both plant and animal hosts $[14,18]$. The remaining 38 single-copy orthologues share an average amino acid identity (AAI) of $61.13 \%$ among the 461 enterobacteria with complete flag-2 loci. 
Table 1 Proportion of Enterobacterales families and genera where flag-2 loci are present

\begin{tabular}{|c|c|c|c|c|}
\hline & \# strains & $\%$ containing flag-2 locus & \# genera/species & $\%$ Genera/species with flag-2 loci \\
\hline Budviciaceae & 9 & $78 \%$ & 4 & $75 \%$ \\
\hline Budvicia & 2 & $100 \%$ & 1 & $100 \%$ \\
\hline Leminorella & 2 & $50 \%$ & 2 & $50 \%$ \\
\hline Limnobaculum & 1 & - & 1 & - \\
\hline Pragia & 4 & $100 \%$ & 1 & $100 \%$ \\
\hline Enterobacteriaceae & 2464 & $13 \%$ & 32 & $53.13 \%$ \\
\hline Atlantibacter & 5 & - & 2 & - \\
\hline Buttiauxella & 8 & $38 \%$ & 7 & $28.57 \%$ \\
\hline Cedeceae & 8 & $75 \%$ & 7 & $71.42 \%$ \\
\hline Citrobacter Clade A & 157 & $58 \%$ & 10 & $80.00 \%$ \\
\hline Citrobacter Clade B & 21 & $24 \%$ & 4 & $75.00 \%$ \\
\hline Citrobacter Clade C & 4 & $100 \%$ & 1 & $100.00 \%$ \\
\hline Citrobacter Clade D & 30 & $100 \%$ & 1 & $100.00 \%$ \\
\hline Cronobacter & 189 & - & 7 & - \\
\hline Enterobacter & 608 & $5 \%$ & 28 & $21.43 \%$ \\
\hline Escherichia & 522 & $24 \%$ & 8 & $87.50 \%$ \\
\hline Franconibacter & 10 & - & 2 & - \\
\hline Klebsiella Clade A & 100 & - & 1 & - \\
\hline Klebsiella Clade B & 310 & - & 4 & - \\
\hline Klebsiella Clade C & 189 & - & 3 & - \\
\hline Kluyvera & 9 & $44 \%$ & 6 & $66.67 \%$ \\
\hline Kosakonia & 24 & - & 9 & - \\
\hline Leclercia & 10 & - & 2 & - \\
\hline Lelliottia & 13 & $69 \%$ & 6 & $66.67 \%$ \\
\hline Mangrovibacter & 2 & $100 \%$ & 2 & $100.00 \%$ \\
\hline Metakosakonia & 3 & $33 \%$ & 1 & $33.33 \%$ \\
\hline Phytobacter & 2 & - & 2 & - \\
\hline Pluralibacter & 17 & $12 \%$ & 1 & $100.00 \%$ \\
\hline Pseudescherichia & 2 & $100 \%$ & 1 & $100.00 \%$ \\
\hline Pseudocitrobacter & 1 & $100 \%$ & 1 & $100.00 \%$ \\
\hline Raoultella & 85 & - & 4 & - \\
\hline Salmonella & 112 & - & 2 & - \\
\hline Shimwellia & 2 & - & 1 & - \\
\hline Siccibacter & 6 & $33 \%$ & 2 & $50 \%$ \\
\hline Superficieibacter & 2 & - & 1 & - \\
\hline Trabulsiella & 8 & - & 3 & - \\
\hline Yokenella & 5 & - & 1 & - \\
\hline Erwiniaceae & 287 & $2 \%$ & 8 & $50 \%$ \\
\hline Buchnera & 58 & - & 42 & - \\
\hline Erwinia & 63 & $3 \%$ & 17 & $12 \%$ \\
\hline Izhakiella & 2 & $100 \%$ & 2 & $100 \%$ \\
\hline Mixta & 4 & - & 4 & - \\
\hline Pantoea & 151 & $1 \%$ & 25 & $8 \%$ \\
\hline Phaseolibacter & 1 & - & 1 & - \\
\hline
\end{tabular}


Table 1 Proportion of Enterobacterales families and genera where flag-2 loci are present (Continued)

\begin{tabular}{|c|c|c|c|c|}
\hline & \# strains & $\%$ containing flag-2 locus & \# genera/species & $\%$ Genera/species with flag-2 loci \\
\hline Rosenbergiella & 1 & $100 \%$ & 1 & $100.00 \%$ \\
\hline Tatumella & 5 & $20 \%$ & 4 & $25 \%$ \\
\hline Wigglesworthia & 2 & - & 2 & - \\
\hline Hafniaceae & 97 & $29 \%$ & 4 & $50 \%$ \\
\hline Edwarsiella & 50 & - & 5 & - \\
\hline Enterobacillus & 1 & - & 1 & - \\
\hline Hafnia & 42 & $57 \%$ & 3 & $100 \%$ \\
\hline Obesumbacterium & 4 & $100 \%$ & 1 & $100 \%$ \\
\hline Morganellaceae & 313 & $0 \%$ & 7 & $0 \%$ \\
\hline Arsenophonus & 2 & - & 1 & - \\
\hline Moellerella & 2 & - & 1 & - \\
\hline Morganella & 55 & - & 5 & - \\
\hline Photorhabdus & 31 & - & 9 & - \\
\hline Proteus & 122 & - & 14 & - \\
\hline Providencia & 58 & - & 14 & - \\
\hline Xenorhabdus & 43 & - & 24 & - \\
\hline Pectobacteriaceae & 244 & - & 7 & - \\
\hline Biostraticola & 1 & - & 1 & - \\
\hline Brenneria & 9 & - & 6 & - \\
\hline Dickeya & 55 & - & 9 & - \\
\hline Lonsdalea & 35 & - & 4 & - \\
\hline Pectobacterium & 140 & - & 16 & - \\
\hline Samsonia & 1 & - & 1 & - \\
\hline Sodalis & 3 & - & 3 & - \\
\hline Thorselliaceae & 1 & - & 1 & $0 \%$ \\
\hline Thorsellia & 1 & - & 1 & - \\
\hline Yersiniaceae & 605 & $37 \%$ & 12 & $25.00 \%$ \\
\hline Chania & 2 & $50 \%$ & 2 & $50 \%$ \\
\hline Ewingella & 2 & - & 1 & - \\
\hline Gibsiella & 4 & - & 1 & - \\
\hline Nissabacter & 1 & - & 1 & - \\
\hline Rahnella & 18 & - & 7 & - \\
\hline Rouxiella & 3 & $33 \%$ & 3 & $33.33 \%$ \\
\hline Serratia Clade A & 159 & - & 10 & - \\
\hline Serratia Clade B & 12 & - & 1 & - \\
\hline Serratia Clade C & 8 & - & 2 & - \\
\hline Serratia Clade D & 1 & - & 1 & - \\
\hline Serratia Clade E & 1 & - & 1 & - \\
\hline Yersinia & 394 & $56 \%$ & 24 & $62.50 \%$ \\
\hline Family Unassigned & 8 & $100 \%$ & 1 & $100 \%$ \\
\hline Plesiomonas & 8 & $100 \%$ & 1 & $100 \%$ \\
\hline Overall & 4028 & $15 \%$ & 72 genera & $43.06 \%$ \\
\hline
\end{tabular}

The families in the order Enterobacterales incorporated in this study, and the prevalence of flag-2 loci among them are indicated in bold 


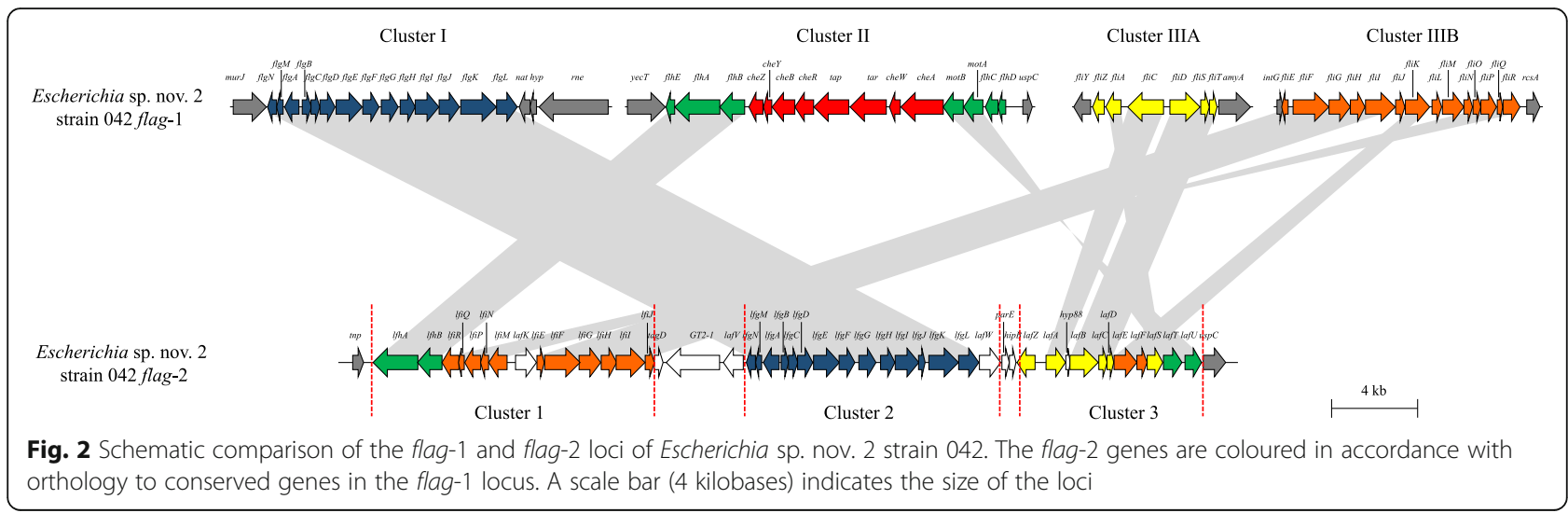

In accordance with the study on the flag-2 locus of Escherichia sp. nov. 2 strain 042, the flag-2 loci can be subdivided into three distinct gene clusters - Cluster 13 (Fig. 2) [15]. Cluster 1, comprised of fourteen genes lfhAB-lfiRQPNM-lafK-lfiEFGHIJ, encodes the proteins involved in regulation and assembly of the basal body components and is analogous to the flhAB-fliRQPNMEFGHIJ genes in the flag-1 locus (Fig. 3) [7, 15]. The encoded orthologues among the 461 complete complement strains share $67.78 \%$ AAI. One Cluster 2 protein restricted to the flag-2 loci, LafK, has been predicted to serve as regulator of flagellum biosynthesis [15] and shares $67.23 \%$ AAI among the 461 strains with complete flag-2 loci. Cluster 2 also typically comprises fourteen genes, lfgNMABCDEFGHIJKL, which are orthologous to flgNMABCDEFGHIJKL in the flag-1 locus and encode flagellar structural proteins (Fig. 3) [12]. The flag-2 cluster 2 proteins show slightly greater variability than the cluster 1 genes, sharing 61.44\% AAI, with four proteins, LfgN (chaperone), LfgM (Anti $\sigma^{28}$ factor), LfgA (basal body P-ring protein) and LfgL (hook-associated protein) sharing $<50 \%$ AAI.

Cluster 3 comprises of the genes lafWZABCDEFSTU, which code for eleven proteins with substantially lower

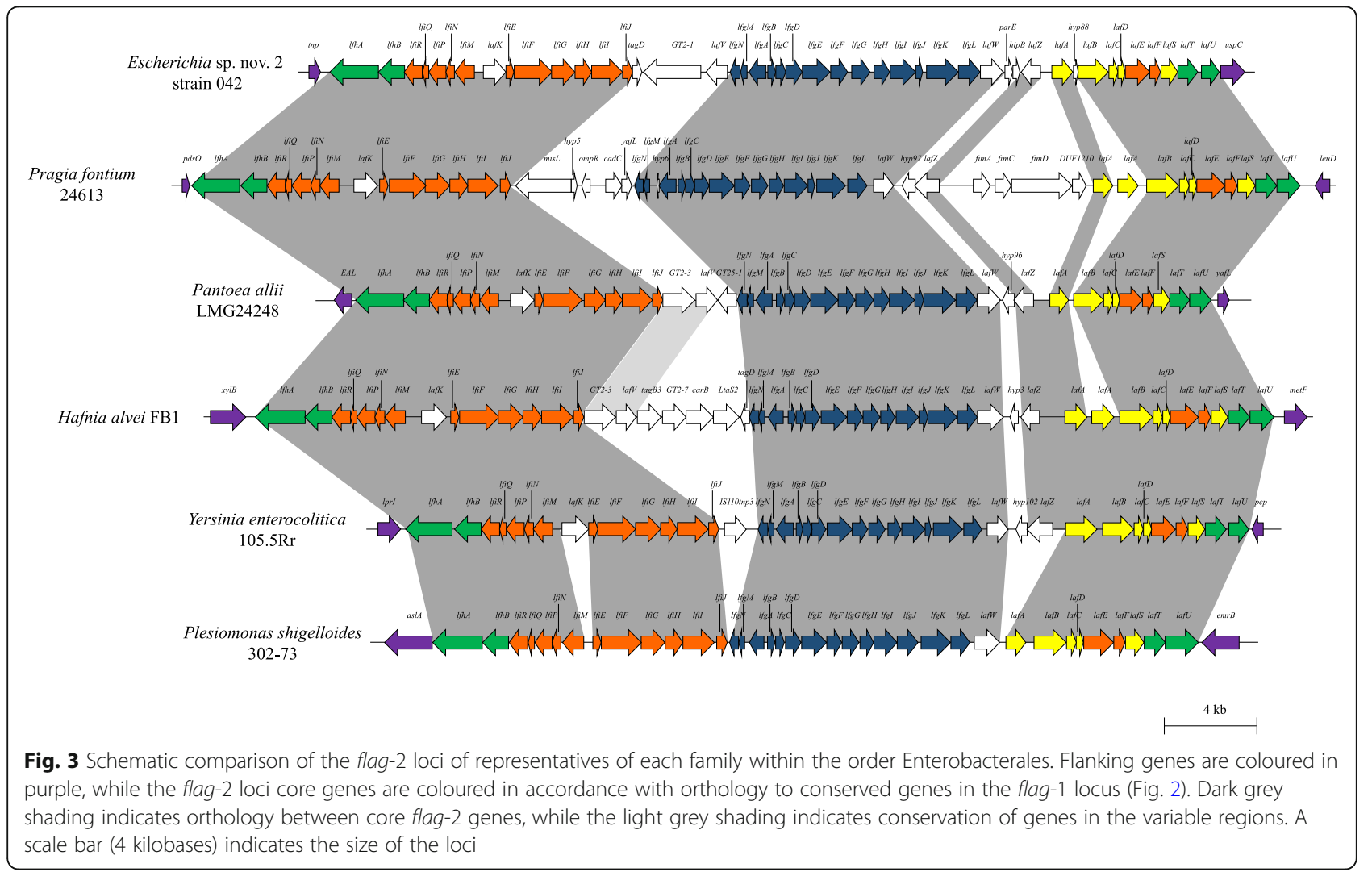


orthology (50.07\% AAI) than those in Cluster 1 and 2. These include proteins involved in filament synthesis (LafABCD - orthologues of FliCDST), $\sigma^{28}$ factor LafS (orthologue of FliA) and the motor proteins LafT and LafU (orthologues of MotA and MotB in the flag-1 locus) (Fig. 3). Also within this cluster are genes coding for the proteins LafW and LafZ, which represent a putative hook-associated protein and transmembrane regulator, respectively [15], orthologues of which are absent from the flag-1 locus. The latter proteins share lower AAI values of 44.57 and $38.89 \%$, respectively.

\section{Gene and en bloc deletion may have resulted in non- functionality of the flag-2 system in some Enterobacterales taxa}

While a substantial fraction of the flag-2 loci contain a complement of 39 conserved genes coding for proteins involved in flagellar biosynthesis and functioning, $22.13 \%$ of enterobacterial strains are missing at least one of these genes. For example, 22/67 Y. enterocolitica strains are missing the entire Cluster 1 (lfhABlfiRQPNM-lafK-lfiEFGHIJ), while 3/91 Citrobacter Clade A strains lack both Cluster 2 and Cluster 3. Transposition appears to be a major driver of the observed en bloc gene deletions. As such, twenty-five distinct transposase genes are localised within the Enterobacterales flag-2 loci. These belong to a range of different transposase families, including IS1, IS4, IS5, IS110 and Mu transposases and are integrated in diverse locations within the flag-2 loci. The reading frames of individuals genes could also be observed to be disrupted by transposase integration, with lfgF (20 strains) and lfiG (7 strains), being particularly prone.

Previous analyses showed that in many Escherichia and Shigella strains, a deletion has occurred within the reading frames of the $l f h A$ and lafU genes which occur at the $5^{\prime}$ and $3^{\prime}$ ends of the flag-2 locus, respectively, resulting in loss of the remaining locus between the lfh $A$ and lafU pseudogene fragments. The presence of direct repeats at the ends of this deletion suggest that this may have resulted through recombination events [15]. Blast analyses of the $l f h A$ and laf $U$ genes and proteins against the 4028 Enterobacterales strains showed that this occurs in the genomes of $531(13.18 \%)$ of the strains (Fig. 1 - indicated by blue triangles). The lfhA and lafU pseudogenes are primarily found in those taxa where complete flag-2 loci are present. For example, of the 100 E. coli strains analysed, all 76 strains that lack flag-2 loci contain the truncated gene copies. Similarly, 50 (75.76\%) of the 66 Citrobacter Clade A strains lacking flag-2 loci show evidence of its deletion. This suggests that the flag-2 locus is likely to have been a far more prevalent feature among the Enterobacterales (27.88\%; 1123/4028 analysed strains) prior to en bloc deletion of the locus in a substantial number of strains. While large scale deletions are partially responsible for the difference in size and protein complement observed among the enterobacterial flag-2 loci, it can further be attributed to the integration of a substantial set of non-conserved cargo genes within the loci.

\section{The enterobacterial flag-2 loci are hotspots for integration of cargo genes}

Alignment of the enterobacterial flag-2 loci and comparative analysis of their encoded protein complements revealed that, although extensive synteny and a substantial set of conserved proteins occur among these loci (Fig. 2), there are 349 distinct protein coding genes, which are not conserved among all enterobacterial flag-2 loci and which do not form part of the core set involved in flagellar biosynthesis and functioning. As such, they can be considered as cargo genes within the flag-2 loci. A substantial proportion (121 genes; $34.67 \%$ of cargo genes) of these genes code for hypothetical proteins and proteins containing domains of unknown function. However, BlastP searches against the NCBI nonredundant protein database and the Conserved Domain Database [19], identified proteins with a range of nonflagellar related functions within the flag-2 loci. For example, the flag-2 loci of twenty-one Escherichia strains incorporate genes coding for the restriction endonuclease EcoRII (pfam09019; E-value: 8.36E-98; Average size: 401 aa; AAI: $97.1 \%)$ and DNA cytosine methylase Dcm (PRK10458; E-value: 0.0; Average size: 474 aa; AAI: 98.6\%). These function in cleaving DNA at a specific sequence and methylation of this sequence to prevent restriction and protect the bacterial cell from integration of bacteriophage and plasmid DNA [20]. Four Pragia fontium strains incorporate genes coding for the pilin protein FimA (PRK15303; E-value: 4.13E-03), periplasmic chaperone FimC (PRK09918; E-value: 3.07E-91) and usher protein FimD (PRK15304; E-value: 0.0).

Cargo genes are found interspersed throughout the flag-2 loci, usually in single or two gene clusters. However, two regions appear to be particularly prone to integration of cargo genes. The first variable region (VR1) occurs between the flag-2 gene clusters 1 and 2 (between lfiJ and $l f g N$ ), while the second (VR2) occurs at the $5^{\prime}$ end of cluster 3 (between lafW and lafZ) (Fig. 2). VR1 occurs in the flag-2 loci of 382/592 (64.53\%) enterobacterial strains and is particularly prevalent in members of the family Budviciaceae (7/7 strains), Enterobacteriaceae (310/ 316 strains) and Hafniaceae (28/28 strains), but are more restricted among the flag-2 loci of the Erwiniaceae (4/8 strains) and Yersiniaceae (33/225 strains; 14.67\%). The VR1 regions vary in size between 0.7 and $18.9 \mathrm{~kb}$ (average size: $5.9 \mathrm{~kb}$ ) and code for between one and twenty-three (average proteins: 5) proteins (Additional file 1: Table S3). 
This region shows evidence of having been derived through horizontal gene transfer, with $\mathrm{G}+\mathrm{C}$ content deviations of -15.1 to $+4.1 \%$ (average $-2.6 \%$ ) and -12.5 to + $4.2 \%$ (average $-3.6 \%$ ) from the genomic and flag- $2 \mathrm{G}+\mathrm{C}$ content, respectively. A total of 154 distinct proteins are encoded by the VR1 regions of the enterobacterial flag-2 loci. VR2 is more prevalent compared to VR1, occurring in 478/592 (80.74\%) of all Enterobacterales. This includes the flag-2 loci of all Budviciaceae and Hafniaceae and 93.78, 72.47 and $37.5 \%$ of the Yersiniaceae, Enterobacteriaceae and Erwiniaceae, respectively. This variable region is typically smaller than VR1, ranging in size from 0.4 to $5.5 \mathrm{~kb}$ (average size: $0.6 \mathrm{~kb}$ ) and coding for between one and six (average: 1 protein) distinct proteins. This region also shows evidence of horizontal acquisition, with an even more pronounced $\mathrm{G}+\mathrm{C}$ deviation of -13.6 to + $8.7 \%$ (average: $-4.7 \%$ ) and -15.4 to $+5.1 \%$ (average: $4.7 \%$ ) from the genomic and flag-2 locus $\mathrm{G}+\mathrm{C}$ contents, respectively. VR2 codes for twenty-seven distinct proteins, with the majority of these $(21 / 27 ; 77.78 \%)$ being hypothetical proteins or those containing domains of unknown function (Additional file 1: Table S3). By contrast, many of the proteins in VR1 share orthology with proteins involved in glycosylation and modification of the flagellar filament.

\section{The flag-2 variable region 1 (VR1) encodes the machinery for glycosylation, methylation and modification of the flagellum}

The most prevalent genes among the (520/1826 total VR1 proteins; 28.48\%) enterobacterial flag-2 VR1 regions are those that code for twenty-two distinct glycosyltransferases. Glycosyltransferase enzymes catalyze glycosidic bond synthesis resulting in the covalent attachment of a glycan to proteins or other sugars and are likely to contribute towards glycosylation of the main structural protein of the flagellum, flagellin $[14,15]$. This posttranslational modification has been linked to a wide range of phenotypes, including surface recognition, adhesion, biofilm formation, antigen masking from immune response and virulence $[14,15]$. Flagellin glycosylation is a relatively common feature among the Enterobacteriaceae and the flag-1 flagellar systems of $307 / 2000$ enterobacteria (15.4\%) were predicted to be glycosylated [14]. Here, $341 / 592(57.60 \%)$ of the flag-2 loci contain genes coding for glycosyltransferases which are predicted to be involved in flagellin glycosylation. BlastP comparison against the CAZy database, using the dbCAN pipeline [21], classified these flag-2 proteins into five distinct glycosyltransferase families. Ten distinct proteins belong to the GT2 family (GT2-1 to GT2-10), members of which transfer a wide array of saccharides including mannose, galactose, $\mathrm{N}$-acetylglucosamine, glucose and their derivatives $[14,22]$. The most common of these GT2 glycosyltransferases is GT2-1, which occurs in 222 Enterobacteriaceae belonging to eight distinct genera. The VR1 of seventeen strains in six genera of Enterobacteriaceae and Erwiniaceae incorporate a gene coding for a glycosyltransferase of the GT4 family, which likewise transfer a broad range of sugars, including glucose, mannose and glucosamine [22]. A further 70 strains encode five distinct glycosyltransferase orthologues (GT9-1 to GT9-5) of the GT9 family, which incorporates lipopolysaccharide $\mathrm{N}$-acetylglucosaminyltransferases and heptosyltransferases [22]. Another represented glycosyltransferase family, GT25 comprises four distinct orthologues (GT251 to GT25-4) in a total of twenty-six taxa and includes galactosyltransferases and proteins involved in lipopolysaccharide biosynthesis. Finally, fourteen strains incorporate two different types of GT32 (GT32-1 and GT32-2) family glycosyltransferases with a purported role in mannose, galactose and glucosamine saccharide transfer [22].

Given that distinct sugars can be incorporated by glycosyltransferases belonging to the different GT families, the type of sugars incorporated in the flagellin glycan cannot solely be determined on the basis of the type of glycosyltransferase present. Proteins that catalyse the synthesis of these sugar moieties may be localized in genomic locations other than the flag-2 locus. However, the VR1 region of 35 Citrobacter Clade A strains encodes orthologues of the sialyltransferase PM0188 (pfam11477; E-value: 2.54E-16) of Pasteurella multocida, suggesting that the flagellin glycan of the latter strains may incorporate neuraminic acid [23]. Orthologues of lipoteichoic acid synthase LtaS (LtaS1 and LtaS2; cd16015; 30 strains across three genera in the Enterobacteriaceae and Hafniaceae) and CDP-glycerol phosphotransferase TagB (TagB1 to TagB8; COG1887; 65 strains across thirteen genera in the Enterobacteriaceae and Hafniaceae) are encoded on the VR1 regions of flag-2 loci. Additionally this region encodes a glycerol-3phosphate cytidylyltransferase TagD (cd02171; E-value: 4.09E-46) in 235 strains across ten genera and three families. These proteins are central to the synthesis of teichoic acid, phosphodiester-linked polyol glycopolymers that form a major part of the cell wall of most Gram-positive bacteria [24]. The presence of orthologues of genes involved in this function in VR1 suggest that these glycopolymers form part of the flagellin glycan of the enterobacterial flag-2 system.

While the exact glycan sugar moieties of the flagellin glycans are difficult to determine, the presence of orthologues of diverse proteins which may modify or substitute the glycan chains suggest the flag-2 flagellin glycan is heavily decorated as has been observed in the primary flagellar system of many enterobacteria as well as Grampositive bacteria $[14,18]$. Among the VR1-encoded proteins are orthologues of the acetyltransferases NeuD 
(Neuraminic acid acetyltransferase; TIGR03570; E-value: 2.65E-61; Citrobacter Clade A werkmannii AK-8), RimI (N-acetyltransferase; pfam00583; E-value: 1.69E-11; Escherichia sp. nov 1 E1642), OptS (O-phosphoseryl acetylase; PRK06253; E-value: 5.94E-03; two Escherichia albertii strains) and $\mathrm{WbbJ}$ (maltose $\mathrm{O}$-acetyltransferase; cd04647; E-value: 3.33E-25; twelve Citrobacter Clade A strains), the pyruvyl transferase WcaK (pfam04230; Evalue: 2.59E-16; fourteen Citrobacter Clade A strains) and transaminase WecE (dTDP-4-amino-4,6-dideoxygalactose transaminase; COG0399; E-value: 4.48E-103; twenty-nine Enterobacteriaceae and Hafniaceae strains). Furthermore, fifteen distinct methyltransferases belonging to the FkbM (FkbM1 to - 3; TIGR01444), Mtf11 (Mtf11-1-3; pfam08241), Mtf12 (pfam08242), Mtf23 (Mtf23-1 to - 4; pfam13489), Mtf24 (Mtf24-1 and - 2; pfam13578) and Mtf25 (Mtf25-1 and - 2; pfam13649) families are encoded in the flag-2 VR1 regions. This suggests that the flag-2 flagellin glycans are decorated with methyl, acetyl, amino and pyruvyl groups. An additional $N$-lysine methylase FliB has been observed to be relatively common among the flag-1 loci and flagellin methylation has been suggested to play a role in virulence in Salmonella enterica [14, 25]. Orthologous proteins, previously termed LafV (LafV1 to LafV10), occur in the flag-2 VR1 regions of 342/592 (57.58\%) enterobacteria advocating that flagellin methylation is also a common phenomenon in the flag-2 flagellar system. While a range of functions have been ascribed to flagellin glycosylation and methylation for the primary flagellar system, its prevalence among the flag-2 loci suggests similar important roles in the latter system, but this needs to be confirmed experimentally.

\section{The enterobacterial flag-2 locus displays a complex evolutionary history of vertical transmission and horizontal acquisition}

The relatively low prevalence of flag-2 loci among the Enterobacterales suggests that they may have been acquired through horizontal gene transfer (HGT) in a select set of taxa. Comparison of a phylogeny on the basis of 32 flag- 2 proteins conserved among 87 taxa representative of six families and 27 genera and a housekeeping protein phylogeny shows that there is congruence at the genus and family level for some taxa (Fig. 4). For example, Yersinia and Rouxiella (Yersiniaceae), Hafnia and Obesumbacterium (Hafniaceae) and Pragia and Budvicia (Budviciaceae) form cohesive clades in both phylogenies (Fig. 4). This suggests the flag-2 loci have been maintained via vertical transmission through speciation events in these evolutionary lineages. However, a more complex evolutionary history can be ascribed to the flag-2 loci of some taxa. The flag-2 locus of Leminorella grimontii ATCC 33999 (Budviciaceae) clusters with those of the Enterobacteriaceae Citrobacter Clade A and C and Escherichia, while that of Chania multitudinisentens RB-25 clusters with Pluralibacter (Enterobacteriaceae). Similarly, the flag-2 loci of Pantoea brennerii IF5SW-P1 and Pantoea allii LMG 24248 cluster distinctly with Enterobacter and Lelliottia spp. and not with the other taxa, Erwinia and Izhakiella, of the Erwiniaceae. Moreover, the Enterobacteriaceae form three distinct clades when considering their flag-2 loci (Fig. 4). This suggests that flag-2 loci have been derived through HGT events in some of these taxa. Genomic regions derived through recent HGT events are often typified by $\mathrm{G}+\mathrm{C}$ contents that vary substantially from the rest of the genome [26]. In general, the flag-2 loci of all 592 enterobacterial strains have a $\mathrm{G}+\mathrm{C}$ content only marginally above (average $\mathrm{G}+\mathrm{C}$ deviation $=0.5 \%$; range: 4.0 to $+5.0 \%$ ) that of the remainder of the genome (Additional file 1: Table S2). This may be attributed in part to the distinct $\mathrm{G}+\mathrm{C}$ content of the VR1 and VR2 regions. Exclusion of these regions, however, resulted in even more pronounced $\mathrm{G}+\mathrm{C}$ deviations for the flag-2 loci (average $\mathrm{G}+\mathrm{C}$ deviation: $+1.1 \%$; range: -4.0 to $+5.8 \%$ ) suggesting horizontal acquisition in a more substantive set of taxa. This is particularly evident in the Enterobacteriaceae, where the flag-2 loci have an average $\mathrm{G}+\mathrm{C}$ content $2.1 \%$ (range -3.1 to + $5.8 \%$ ) above that of the rest of the genome. It is furthermore evident in those taxa placed in distinct family clades in the flag- 2 tree, including $L$. grimontii ATCC 33999 (G + C deviation: + 2.5\%), C. multitudinisentens $\mathrm{RB}-25(\mathrm{G}+\mathrm{C}$ deviation: $+2.4 \%)$ and $P$. allii LMG 24248 ( $G+C$ deviation: - 3.6\%). In one of the Enterobacteriaceae clades in the flag- 2 tree, $\mathrm{G}+\mathrm{C}$ deviations are pronounced in Escherichia $(\mathrm{G}+\mathrm{C}$ deviation: + 4.8\%), Pseudocitrobacter ( $\mathrm{G}+\mathrm{C}$ deviation: + $2.8 \%$ ) and Siccibacter (-2.9\%). By contrast, the flag-2 loci in taxa belonging to Citrobacter Clade A have average $\mathrm{G}+\mathrm{C}$ deviations of $0.0 \%$, suggesting that the latter may represent ancestral flag-2 loci which have been derived through HGT in the former strains. However, among the flag-2 loci of Escherichia spp. (124 strains) and Yersinia spp. (223 strains) G + C deviations of +2.6 to $+5.8 \%$ and -3.0 to $+1.0 \%$ could be observed, indicating complex evolutionary histories for the flag-2 loci even within these apparently stable lineages.

\section{The flag-2 flagellar system may represent a multi- functional machine among the Enterobacterales}

A broad range of functions have been ascribed to the flag-1 flagellar system, including swimming and swarming motility, adhesion, biofilm formation, host invasion and colonization [11]. By contrast, little is known about the function(s) of the secondary flagellar system among the Enterobacterales. The deletion (between $l f h A$ and 


\section{flag-2 protein tree}

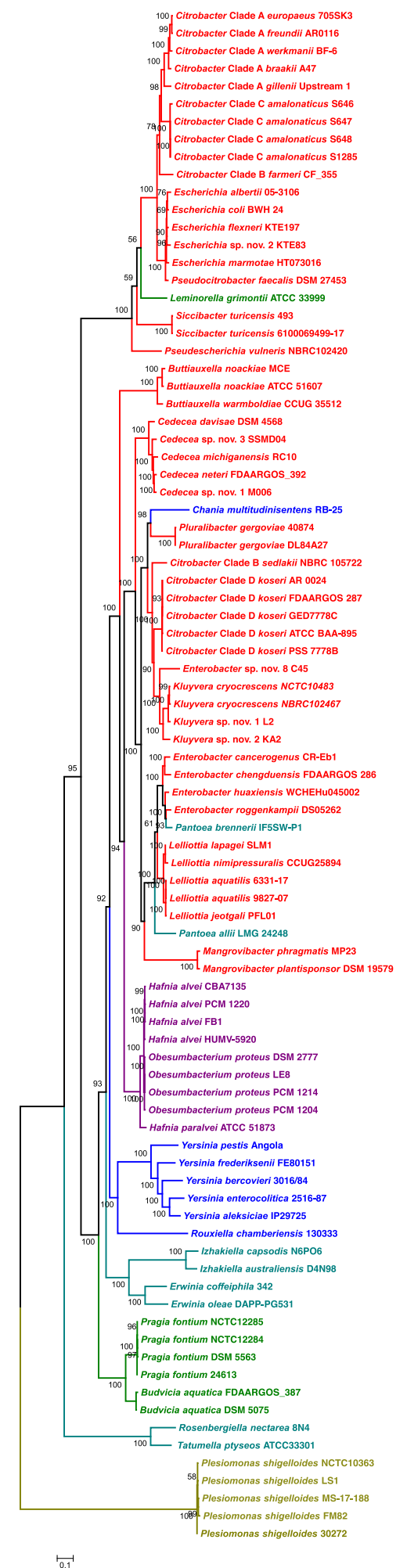

$\stackrel{1}{0.1}$

Fig. 4 (See legend on next page.)

\section{House-keeping protein tree}

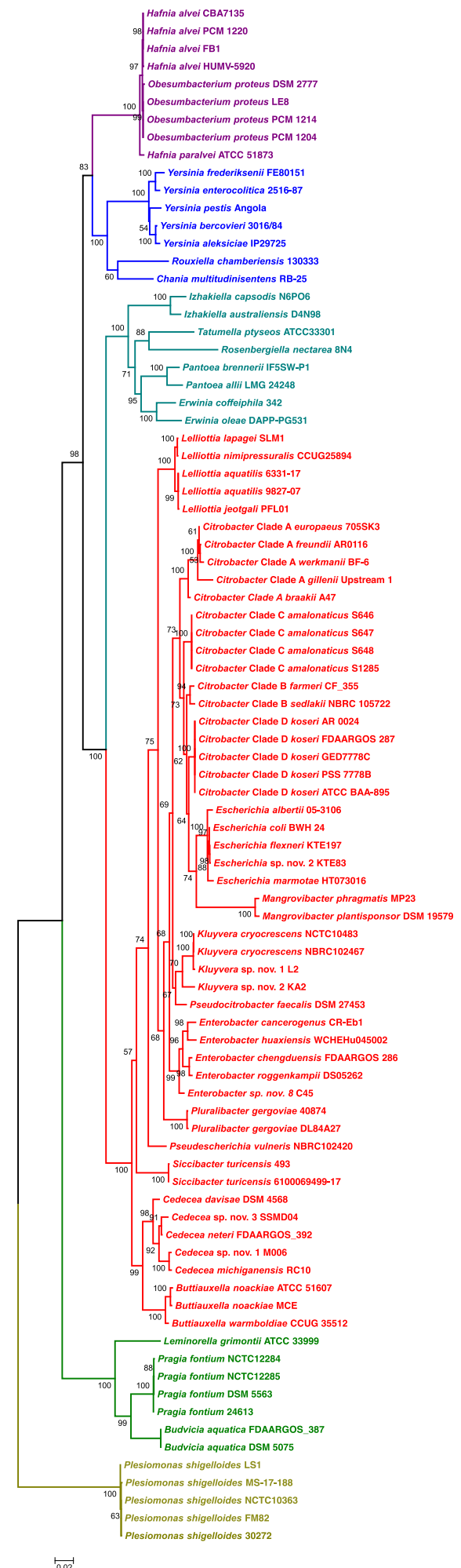


(See figure on previous page.)

Fig. 4 Comparison of conserved flag-2 protein tree and house-keeping protein tree. A ML phylogeny was constructed on the basis of a concatenated alignment of 32 conserved flag-2 proteins (left) as well as on the basis of the house-keeping proteins GyrB, InfB, RecA and RecB (InfB). The final alignments for the flag-2 protein tree and house-keeping proteins comprised of 5800 and 2893 amino acid sites, respectively. The best-fit evolutionary models $L G+F I+G 4$ were used for construction of both phylogenies. Bootstrap values ( $n=1000$ replicates) are indicated. The strains and branches are coloured according to the family to which each belong

laf(U) in Escherichia or frameshift mutation in Escherichia sp. nov. 2 strain 042 was deemed to inactivate the flag-2 system, rendering it non-functional [15]. Phage integration within both the flag-1 and flag-2 loci of $C$. rodentium has been suggested to have resulted in their non-motile phenotype [27]. This is in line with the concept that synthesis and rotation of the primary (flag-1) system, represents a significant metabolic burden on the bacterial cell, accounting for $2.1 \%$ of the overall energy requirement in $E$. coli, where the flag-2 system may not provide a selective advantage to the cell $[15,28]$. However, several lines of evidence suggest the flag-2 system plays several important roles in other enterobacterial taxa. The flag-2 flagella of Plesiomonas shigelloides have been shown to be essential for swarming motility [29]. Here we have observed flag-2 loci in the genomes of members of the family Budviciaceae (two strains of Budvicia aquatica and four strains of P. fontium) and Erwiniaceae (Tatumella pytseos ATCC 33301), which lack the flag-1 flagellar system but have nevertheless been described as being capable of flagellar motility [2]. By contrast, L. grimontii ATCC 33999 (Budviciaceae) and Rouxiella chamberiensis 13,033 (Enterobacteriaceae), which lack a flag-1 locus but retain a flag-2 locus, have been described as non-motile [2]. The flag-2 locus of the latter strain is, however, missing the lafZ gene.

While the flag-2 loci of many of the enterobacterial taxa contain deletions or genes with disrupted reading frames, a substantial number $(77.87 \%$ of strains containing flag-2 loci) appear to encode the full complement of proteins for the synthesis and functioning of this flagellar system. Many of these have functional flag-1 loci, and as such the flag-2 system may play roles other than in motility in these taxa. The flag-1 system has been implicated in the secretion of virulence factors by pathogenic bacteria, including the phospholipase YplA in $Y$. enterocolitica, the lipase XlpA and antibacterial xenocin in the entomopathogen Xenorhabdus and invasion antigen (Cia) protein in Campylobacter spp. [4, 30, 31]. Analysis of the proteins encoded in the VR1 and VR2 regions of the flag-2 loci revealed a number of putative secretion targets among the cargo proteins. The VR1 region of the Escherichia fergusonii YH17130 flag-2 locus incorporates a gene coding for a Type VI secretion system (T6SS) effector protein VgrG (COG3501; E-value: $0.0)$. This gene is flanked by genes coding for a 1556 amino acid Rhs-domain containing protein (COG3209;
E-value: $8.53 \mathrm{E}-44$ ) and polymorphic toxin immunity protein Imm26 (pfam15428; E-value: 1.7E-15). Rhs-domain proteins represent toxins that have been found to be involved in inter-bacterial competition and are also secreted via the T6SS, where the Imm 26 protein may serve as a protective mechanism against autotoxicity [32]. Furthermore, the VR1 and VR2 regions in the flag-2 locus of two and three Lelliottia spp., respectively encode orthologues of two distinct Haemolysin coregulated (Hcp) proteins (Hcp1; pfam05638; E-value: 1.48E-30; Hcp2; COG3157; E-value: 1E-40), which likewise serve as secretion effector proteins [33]. The presence of distinct secreted effector proteins in a number of strains imply a putative role for the flag-2 system in secretion. Furthermore, within the VR1 regions of the Escherichia sp. nov. 1 strain E1642 and Lelliottia aquatilis 6331-17 are genes coding for an orthologue of pesticin (Pst; CD16903; E-value: 1.88E-73), a phage lysozyme-like bacteriocin from $Y$, pestis and an orthologue of the RNase toxin Ntox44 (pfam15607; E-value: 4.52e-16) [34, 35], respectively, ascribing a role in antibacterial activity or inter-bacterial competition to the flag-2 system. Finally, four P. fontium (Budviciaceae) incorporate a gene coding for an 879 aa orthologue $(96.6 \%$ AAI) of the autotransporter MisL (PRK15313; E-value: 6.51E-97). In S. enterica serovar Typhimurium this protein plays a role in adherence, aggregation and biofilm formation [36].

\section{Discussion}

The flag-2 locus is a relatively prevalent phenomenon among the Enterobacterales, comprising of a syntenous locus coding for 39 well-conserved proteins. Comparative genomic analyses revealed that frequent transposon integration has occurred within single genes in the locus, as well as en bloc deletion between the partial reading frames of $l f h A$ and laf $U$. The en bloc deletions and gene disruptions can be envisaged to have a detrimental effect on functionality of the flag-2 system, as has been observed in Escherichia sp. nov 2 strain 042 [15]. Furthermore, pervasive deletion of the locus suggests that it may have been a far more common genomic feature and hints at a complex evolutionary history for the locus. A recent study has identified orthologous flag-2 loci among members of the alpha-proteobacterial order Rhizobiales and furthermore, they are similar to the lateral flagellar system of Vibrio and Aeromonas spp. [15, 37]. A more 
comprehensive analysis across a broader taxonomic scope may shed further light on the evolution of the flag-2 system.

Phylogenetic analysis on the basis of the conserved proteins encoded on the flag-2 loci suggest disparate evolutionary histories for these loci among the enterobacterial taxa, with the flag-2 loci some taxa likely being derived through HGT events, while some lineages shows evidence of retention of this locus through vertical transmission. Analysis of the flag-1 protein complement of forty-one motile species across eleven bacterial phyla showed extensive sequence similarity between the twenty-four core proteins conserved among all taxa [38]. It has thus been postulated that a few genes, or even a single precursor, may have given rise to the full complement of genes required for the synthesis of the primary flagellar system through gene duplications, gene fusions and the recruitment of novel genes [38,39]. It is plausible that similar evolutionary processes may have given rise to the extant flag-2 loci, which may explain the complex evolutionary histories of these loci among the Enterobacterales.

A communal feature of the flag-2 loci of most enterobacterial taxa is the presence of cargo genes, integrated mainly in two variable regions, VR1 and VR2, which mainly encode the machinery for flagellin glycosylation, which as observed in the flag-1 locus, likely represents a common posttranslational feature of this flagellar system. A wide range of functions have been ascribed to this feature in the primary flagellar system [14], but its role in the secondary flagellar system remains to be elucidated. Also among the cargo genes are those which provide hints on the potential functions of the secondary flagellar system. While earlier evidence largely advocated for the non-functionality of the flag-2 system, the elucidation of a functional flag-2 system in P. shigelloides [29] and the presence of flag-2 loci containing the full complement of conserved flagellar biosynthesis and operational genes suggest this may represent a multifunctional system with several potential roles. As many of the taxa with intact flag-2 loci represent both clinical and plant pathogens, knock-out mutagenesis and further characterization are imperative for a deeper understanding of this intriguing flagellar system.

\section{Conclusions}

Highly conserved and syntenous secondary flagellar (flag-2) loci occur in 15\% all screened members of the order Enterobacterales and likely represents important functional features in the taxa that incorporate them. Variable regions within the flag-2 loci code for proteins with roles in posttranslational modification of the flagellar system as well as those that provide hints into the functions of the flag-2 system. Future work will focus on determining the biological role(s) of the secondary flagellar system in members of the order Enterobacterales.

\section{Methods \\ Identification of flag-2 loci among the Enterobacterales}

The genomes of 4028 members of the order Enterobacterales were selected for analysis. Selection was based on the current genomic taxonomy in the Genome Taxonomy DataBase (GTDB Release 04-RS89) [40] and provided coverage of all eight families (75 genera) and Plesiomonas (family unassigned). Up to 100 strains representative of each species were included in the analysis. The presence of flag-2 loci was determined by local tBlastN analyses with the LafK (CAH19120.1), LafW (CAH19144.1), LafZ (CAH19147.1) LafA (CAH19148.1) and LafB (CAH19149.1) proteins of E. coli 042 [15] using BioEdit v.7.2.5 [41]. The lafK, lafW and lafZ genes are conserved among most strains that harbour flag-2 loci and serve as distinguishing factors, as orthologous genes are absent from the flag-1 locus [15]. For those genomes with Blast hits for the above proteins, the complete flag-2 loci were identified by searching the annotated Genbank genome sequences up- and downstream of these genes. These complete flag-2 loci were structural annotated using the Prokaryotic GeneMark.hmm v.2 server [42] and the $\mathrm{G}+\mathrm{C} \%$ contents of each of the loci were calculated using BioEdit v.7.2.5 [41].

\section{Comparative genomic analyses of the enterobacterial flag-2 loci}

Orthologues in the flag-2 loci protein datasets of twentyfive strains were identified using Orthofinder v 1.1.4 [43]. The distinct orthogroups were functionally annotated by BlastP analyses against the NCBI non-redundant (nr) protein database and the NCBI Conserved Domain Database (CDD) using Batch CD-Search [19]. Furthermore, proteins with predicted functions in carbohydrate catabolism were compared against the Carbohydrate Active Enzymes (CAZy) database using the dbCAN server [21] to classify putative glycosyltransferases and glycosidases.

\section{Phylogenetic analyses}

A maximum likelihood (ML) phylogeny of the 4028 enterobacterial strains incorporated in this study was constructed using the concatenated amino acid sequences of four conserved house-keeping proteins, namely Gyrase B (GyrB), translation initiation factor IF2 (InfB), recombinase A (RecA) and RNA polymerase beta subunit (RpoB). The presence/absence/deletion of the flag-2 locus was mapped onto this phylogeny. Similarly a ML phylogeny was constructed on the basis of 32 proteins conserved among 87 enterobacterial taxa representative of the taxonomic spectrum of the order, with up to five strains or species per genus included in the 
analysis. Individual proteins were aligned using the MCoffee implementation of T-Coffee [44]. The aligned proteins were concatenated and poorly aligned blocks were removed using Gblocks v. 0.91b [45]. The curated concatenated alignments were then used to construct ML phylogenies using IQTree v. 1.6.11 [46] using the appropriate evolutionary model predicted using ModelFinder [47] and bootstrap support using UFBoot2 $(n=$ 1000 replicates) [48].

\section{Supplementary information}

Supplementary information accompanies this paper at https://doi.org/10. 1186/s12864-020-6529-9.

Additional file 1: Table S1. Presence/absence of flag-2 loci among 4028 strains belonging to eight families and 72 genera. The presence of flag-1 loci is also indicated. The previous taxonomy denotes the taxonomy according to the NCBI genome database, while the current taxonomy is according to the Genome Taxonomy Database (GTDB). The isolation source as well as habitat/lifestyles of the different strains are given. Table S2. Molecular characteristics of the flag-2 loci among 592 taxa in the Enterobacterales. The sizes of the flag-2 loci, variable regions VR1 and VR2, their $G+C$ contents (\%) and $G+C$ deviation (\%) from the genome are shown. The number of predicted proteins encoded on each of these flag-2 fractions are also shown. Table S3. Characteristics of the cargo genes encoded in the variable regions VR1 and VR2 and elsewhere in the enterobacterial flag-2 loci. The number of strains and the families/ genera in which each protein occurs within the flag-2 loci are indicated, as well as the average amino acid identities among enterobacterial orthologues. Conserved domains present in each cargo protein as determined by BlastP analysis against the Conserved Domain Database are shown

\section{Abbreviations}

AAl: Amino Acid Identity; GT: Glycosyltransferase; HGT: Horizontal Gene Transfer; ML: Maximum Likelihood; Mtf: Methyltransferase

\section{Acknowledgements}

The authors wish to acknowledge the National Research Foundation for student funding (TP).

\section{Authors' contributions}

PDM and TAC conceived the study. PDM and TP performed data analysis. PDM and TP wrote the original manuscript and PDM, TP and TAC contributed to the final version. All authors have read and approved the final manuscript.

\section{Authors' information}

PDM (PhD, Dr) is a Senior Lecturer at the School of Molecular \& Cell Biology, University of the Witwatersrand. TP was a post-graduate student who completed her MSc degree in Microbiology and Biotechnology at the University of the Witwatersrand. TAC (PhD, Prof) is a research leader at the Centre for Microbial Ecology and Genomics, University of Pretoria.

\section{Funding}

TP was funded by the National Research Foundation of South Africa (NRF Masters Scholarship SFH180109299856).

\section{Availability of data and materials}

All genome sequences incorporated in this study are publically available in the NCBI Genome database. The NCBI accession numbers for the contigs/ chromosomes on which the target loci are found are indicated in Additional file 1: Table S1.

Ethics approval and consent to participate Not applicable.
Consent for publication

Not applicable.

\section{Competing interests}

PDM serves as Associate Editor for BMC Genomics. The authors have no other competing interests to declare.

\section{Author details}

${ }^{1}$ School of Molecular \& Cell Biology, University of the Witwatersrand, 2050 Wits, Johannesburg, South Africa. ${ }^{2}$ Centre for Microbial Ecology and Genomics, University of Pretoria 0002, Pretoria, South Africa.

Received: 23 October 2019 Accepted: 22 January 2020

Published online: 30 January 2020

\section{References}

1. Adeolu M, Alnajar S, Naushad S, Gupta RS. Genome-based phylogeny and taxonomy of the 'Enterobacteriales': proposed for Enterobacterales Ord. Nov. divided into the families Enterobacteriaceae, Erwiniaceae fam. Nov. Pectobacteriaceae fam. Nov., Yersiniaceae fam. Nov., Hafniaceae fam. Nov., Morganellaceae fam. Nov., and Budviciaceae fam. Nov. Int J Syst Evol Microbiol. 2016;66:5575-99.

2. Brenner DJ, Farmer JJ III. Family I. Enterobacteriaceae. In: Brenner DJ, Krieg NR, Staley JT, Garrity GM, Boone DR, Vos P, Goodfellow M, Rainey FA, Schleifer KH, editors. Bergey's Manual of Systematic Bacteriology ${ }^{\circledast}$, vol. 2. 2nd ed. Boston: Springer US; 2005. p. 587-607.

3. Jarrell KF, McBride MJ. The surprisingly diverse ways that prokaryotes move. Nat Rev Microbiol. 2008;6:466-76.

4. Duan Q, Zhou M, Zhu L, Zhu G. Flagella and bacterial pathogenicity. J Basic Microbiol. 2013:53:1-8.

5. Haiko J, Westerlund-Wikström B. The role of the bacterial flagellum in adhesion and virulence. Biology (Basel). 2013;2:1242-67.

6. Chevance FF, Hughes KT. Coordinating assembly of a bacterial macromolecular machine. Nat Rev Microbiol. 2008:6:455-65.

7. Bardy SL, Ng SYM, Jarrell KF. Prokaryotic motility structures. Microbiol. 2003; 149:295-304.

8. Samatey FA, Matsunami H, Imada K, Nagashima S, Shaikh TR, Thomas DR, et al. Structure of the bacterial flagellar hook and implication for the molecular universal joint mechanism. Nature. 2004;431:1062-8.

9. Terashima H, Kojima S, Homma M. Flagellar motility in bacteria: structure and function of flagellar motor. Int Rev Cell Mol Biol. 2008;270:39-85.

10. Gao B, Lara-Tejero M, Lefebre M, Goodman AL, Galána JE. Novel components of the flagellar system in Epsilonproteobacteria. mBio. 2014;5:e01349-14.

11. Macnab RM. How bacteria assemble flagella. Ann Rev Microbiol. 2003;57:77-100.

12. Soutourina $\mathrm{OA}$, Bertin PN. Regulation cascade of flagellar expression in gram-negative bacteria. FEMS Microbiol Rev. 2003;27:505-23.

13. Logan SM. Flagellar glycosylation - a new component of the motility repertoire? Microbiol. 2006;152:1249-62.

14. De Maayer P, Cowan DA. Flashy flagella: flagellin modification is relatively common and highly versatile among the Enterobacteriaceae. BMC Genomics. 2016;17:377.

15. Ren CP, Beatson SA, Parkhill J, Pallen MJ. The Flag-2 locus, an ancestral gene cluster, is potentially associated with a novel flagellar system from Escherichia coli. J Bacteriol. 2005:187:1430-40.

16. Bresolin G, Trcek J, Scherer S, Fuchs TM. Presence of a functional flagellar cluster Flag-2 and low-temperature expression of flagellar genes in Yersinia enterocolitica W22703. Microbiol. 2008;154:196-206.

17. Feng L, Liu B, Liu Y, Ratiner YA, Hu B, Li D, et al. A genomic islet mediates flagellar phase variation in Escherichia coli strains carrying the flagellinspecifying locus flk. J Bacteriol. 2008;190:4470-7.

18. De Maayer P, Cowan DA. Comparative genomic analysis of the flagellin glycosylation island of the gram-positive thermophile Geobacillus. BMC Genomics. 2016:17:913

19. Marchler-Bauer A, Derbyshire MK, Gonzales NR, Lu S, Chitsaz F, Geer LY, et al. CDD: NCBI's conserved domain database. Nucleic Acids Res. 2015;43:222-6.

20. Nakayama Y, Kobayashi I. Restriction-modification gene complexes as selfish gene entitites: roles of a regulatory system in their establishment, maintenance, and apoptotic mutual exclusion. Proc Natl Acad Sci U S A. 1998;95:6442-7. 
21. Yin $Y$, Mao X, Yang J, Chen X, Mao F, Xu Y. dbCAN: a web resource for automated carbohydrate-active enzyme annotation. Nucleic Acids Res. 2012;40:W445-51.

22. Coutinho PM, Deleury E, Davies GJ, Henrissat B. An evolving hierarchical family classification for glycosyltransferases. J Mol Biol. 2003;328:307-17.

23. Du K, Yoo JH, Lee YJ, Kim KS, Cho HS. Structural analysis of sialyltransferase PM0188 from Pasteurella multocida complexed with donor analogue and acceptor sugar. BMB Rep. 2008:41:48-54.

24. Schneewind O, Missiakas D. Lipoteichoic acids, phosphate-containing polymers in the envelope of gram-positive bacteria. J Bacteriol. 2014;196: 1133-42.

25. Frye J, Karlinsey JE, Felise HR, Marzolf B, Dowidar N, McClelland M, et al. Identification of new flagellar genes of Salmonella enterica serovar Typhimurium. J Bacteriol. 2006;188:2233-43.

26. Daubin $\mathrm{V}$, Lerat $\mathrm{E}$, Perrière $\mathrm{G}$. The source of laterally transferred genes in bacterial genomes. Genome Biol. 2003:4:R57

27. Magaziner SJ, Zeng Z, Chen B, Salmond GPC. The prophages of Citrobacterium rodentium represent a conserved family of horizontally acquired mobile genetic elements associated with enteric evolution towards pathogenicity. J Bacteriol. 2019;201:e00638-18.

28. Macnab RM. Flagella and motility. In: Neidhardt FC, Curtis III R, Ingraham JL, Lin ECC, Low BK, Magansanik B, Brooks Low K, Schaechter M, Umbarger HE, editors. Escherichia coli and Salmonella: cellular and molecular biology. Washington DC: American Society for Microbiology Press; 1996. p. 123-45.

29. Merino S, Aquilini E, Fulton KM, Twine SM, Tomás JM. The polar and lateral flagella from Plesiomonas shigelloides are glycosylated with legionaminic acid. Front Microbiol. 2015;6:649.

30. McQuade R, Stock SP. Secretion systems and secreted proteins in gramnegative entomopathogenic bacteria: their roles in insect virulence and beyond. Insects. 2018;9:68

31. Young GM, Schmiel DH, Miller VL. A new pathway for the secretion of virulence factors by bacteria: the flagellar export apparatus functions as a protein-secretion system. Proc Natl Acad Sci U S A. 1999;96:6456-61.

32. Ma J, Sun M, Dong W, Pan Z, Lu C, Yao H. PAAR-Rhs proteins harbor various C-terminal toxins to diversify the antibacterial pathways of type $\mathrm{VI}$ secretion systems. Environ Microbiol. 2017;19:345-60.

33. Silverman JM, Agnello DM, Zheng H, Andrews BT, Li M, Catalano CE, et al. Haemolysin coregulated protein is an exported receptor and chaperone of type VI secretion substrates. Mol Cell. 2013;51:584-93.

34. Patzer SI, Albrecht R, Braun V, Zeth K. Structural and mechanistic studies of pesticin, a bacterial homolog of phage lysozymes. J Biol Chem. 2012;287: 23381-96.

35. Zhang D, de Souza RF, Anantharaman V, lyer LM, Aravind L. Polymorphic toxin systems: comprehensive characterization of trafficking modes, processing, mechanisms of action, immunity and ecology using comparative genomics. Biol Direct. 2012;7:18.

36. Wang S, Yang D, Wu X, Wang Y, Wang D, Tian M, et al. Autotransporter MisL of Salmonella enterica serotype Typhimurium facilitates bacterial aggregation and biofilm formation. FEMS Microbiol Lett. 2018;365:fny142.

37. Garrido-Sanz D, Redondo-Nieto M, Mongiardini E, Blanco-Romero E, Durán D, Quelas Jl, et al. Phylogenomic analyses of Bradyrhizobium reveal uneven distribution of the lateral and subpolar flagellar systems, which extends to Rhizobiales. Microorganisms. 2019;7:50.

38. Liu R, Ochman H. Stepwise formation of the bacterial flagellar system. Proc Natl Acad Sci U S A. 2007:104:7116-21.

39. Liu R, Ochman $H$. Origins of flagellar gene operons and secondary flagellar systems. J Bacteriol. 2007:189:7098-104.

40. Parks DH, Chuvochina M, Waite DW, Rinke C, Skarshewski A, Chaumeil PA, et al. A standardized bacterial taxonomy based on genome phylogeny substantially revises the tree of life. Nature Biotechnol. 2018;36:996-1004.

41. Hall TA. BioEdit: a user-friendly biological sequence alignment editor and analysis program for windows 95/98/NT. Nucl Acids Symp Ser. 1999;41:95-8.

42. Besemer J, Lomsadze A, Borodovsky M. GeneMarkS: a self-training method for prediction of gene starts in microbial genomes. Implications for finding sequence motifs in regulatory regions. Nucleic Acids Res. 2001:29:2607-18.

43. Emms DM, Kelly S. OrthoFinder: solving fundamental biases in whole genome comparisons dramatically improves orthogroup inference accuracy. Genome Biol. 2015;16:157.

44. Di Tommaso P, Moretti S, Xenarios I, Orobitg M, Montanyola A, Chang JM, et al. T-coffee: a web server for the multiple sequence alignment of protein and RNA sequences using structural information and homology extension. Nucleic Acids Res. 2011;39:W13-7.

45. Talavera G, Castresana J. Improvement of phylogenies after removing divergent and ambiguously aligned blocks from protein sequence alignments. Syst Biol. 2007:56:564-77.

46. Nguyen LT, Schmidt HA, von Haeseler A, Minh BQ. IQ-TREE: a fast and effective stochastic algorithm for estimating maximum-likelihood phylogenies. Mol Biol Evol. 2015;32:268-74.

47. Kalyaanamoorthy S, Minh BQ, Wong TKF, von Haesler A, Jermiin LS. ModelFinder: fast model selection for accurate phylogenetic estimates. Nat Methods. 2017:14:587-9.

48. Hoang DT, Chernomor O, von Haeseler A, Minh BQ, Vinh LS. UFBoot2: improving the ultrafast bootstrap approximation. Mol Biol Evol. 2018;35: $518-22$.

\section{Publisher's Note}

Springer Nature remains neutral with regard to jurisdictional claims in published maps and institutional affiliations.
Ready to submit your research? Choose BMC and benefit from:

- fast, convenient online submission

- thorough peer review by experienced researchers in your field

- rapid publication on acceptance

- support for research data, including large and complex data types

- gold Open Access which fosters wider collaboration and increased citations

- maximum visibility for your research: over $100 \mathrm{M}$ website views per year

At $\mathrm{BMC}$, research is always in progress.

Learn more biomedcentral.com/submissions 